\title{
Gambling Problems in Patients with Psychotic Disorders in Rural Greece
}

\author{
Roshan Sutar ${ }^{1}$ \\ ${ }^{1}$ Department of Psychiatry, All India Institute of Medical Sciences, \\ Bhopal, India
}

J Neurosci Rural Pract 2020;11:221-221

Gambling problems were defined by authors as: "when patients are repeatedly engaged in gambling and spend disproportionally large amounts, according to their income." ${ }^{1}$ This does not appear to represent "problem gambling" or "pathological gambling" and several factors determining the core features of "problem gambling" are not considered by authors ${ }^{2}$ like relationship disruption, emotional distress, decrements to health, cultural harms, reduced productivity, and criminal activity. However, in light of this discussion gambling as an associated phenomenon appears relevant as per the title of the paper. ${ }^{1}$

Though this study is retrospective, the prevalence of gamblers with schizophrenia (5\%) is in keeping with the literature except those with lifetime history of gambling (7.6\%). ${ }^{1}$ Author has not taken into account other factors that can influence such high numbers. For example, the information is collected from caregivers in the absence of validated questionnaire; therefore, inferring the prevalence of problem gambler in schizophrenia appears as an impetus in the community sample. Five out of six patients received disability pension $^{1}$; whether disability pension was used for gambling is an interesting question that remains unanswered in the discussion.

One of the factors that can influence these numbers include emerging evidence of reward-driven behaviors in schizophrenia that authors should have differentiated from problem gambling, the mention of which appears missing in the article due to the absence of the use of validated questionnaire for gambling. In patients with schizophrenia, reward-driven behaviors, such as an effort to connect with the society and a need for productive activity, could have been misinterpreted in the context of schizophrenia. Due to prefrontal hypodopaminergic state, indulgence into a reward seeking behavior could be mislabeled as "problem gambling" in the modern era where increased tendency to earn through online-offline lottery, sports betting, and card playing may not meet gambling definition per se.

Another factor includes use of antipsychotics, predominantly aripiprazole ${ }^{3,4}$ and flupenthixol, ${ }^{5}$ having causal
Address for correspondence Roshan Sutar, MD Psychiatry, PDF-CLP Department of Psychiatry, AllMS, Bhopal, Madhya Pradesh 462020, India (e-mail: roshidoc@yahoo.co.in).

relationship with gambling-associated behaviors in schizophrenia. The authors have not specified the onset of gambling before, during, or after initiating treatment with antipsychotics keeping in mind the possibility of antipsychotic-induced gambling. Evidence also favors direct exacerbation of psychosis by gambling and a greater involvement in gambling due to the symptoms of schizophrenia. ${ }^{6}$ Perhaps one way of looking at gambling behaviors in schizophrenia could be a secondary phenomenon of spontaneous effort to overcorrect motivation deficit secondary to illness itself or antipsychotics-induced reward behaviors. Considering these factors could have differentiated them from problem gamblers and could have given more reliable numbers. ${ }^{7}$

\section{Conflict of Interest}

None declared.

\section{References}

1 Peritogiannis V, Gioti P, Gogou A. Gambling problems in patients with psychotic disorders in rural Greece Type. J Neurosci Rural Pract 2009;70(9):333-336

2 Langham E, Thorne H, Browne M, Donaldson P, Rose J, Rockloff M. Understanding gambling related harm: a proposed definition, conceptual framework, and taxonomy of harms. BMC Public Health 2016;16:80

3 Lachance A, Corbeil O, Corbeil S, et al. Case reports of aripiprazole and problematic gambling in schizophrenia: a critical review of the evidence. J Clin Psychopharmacol 2019;39 (4):393-397

4 Mété D, Dafreville C, Paitel V, Wind P. Aripiprazole, gambling disorder and compulsive sexuality [in French]. Encephale 2016;42(3):281-283

5 Grötsch P, Lange C, Wiesbeck GA, Lang U. Pathological gambling induced by dopamine antagonists: a case report. J Gambl Stud 2015;31(1):295-297

6 Yakovenko I, Clark CM, Hodgins DC, Goghari VM. A qualitative analysis of the effects of a comorbid disordered gambling diagnosis with schizophrenia. Schizophr Res 2016;171(1-3):50-55

7 Saperia S, Da Silva S, Siddiqui I, et al. Reward-driven decision-makingimpairmentsinschizophrenia.SchizophrRes2019; 206:277-283 